\title{
A contiguous microdeletion syndrome at Xp23.13 with non-obstructive azoospermia and congenital cataracts
}

\author{
Aubrey Milunsky $^{1,2}$ (D) Jeff M. Milunsky ${ }^{1,2} \cdot$ Weilai Dong $^{3} \cdot$ Hayk Hovhannisyan $^{1} \cdot$ Robert D. Oates $^{4}$
}

Received: 30 October 2019 / Accepted: 27 December 2019 /Published online: 9 January 2020

(C) Springer Science+Business Media, LLC, part of Springer Nature 2020

\begin{abstract}
Non-obstructive azoospermia accounts for $10-15 \%$ of male infertility, resulting in $60 \%$ of all cases of azoospermia and affecting about $1 \%$ of the male population. About $30 \%$ of these cases are due to Y chromosome microdeletions, chromosome abnormalities, or hormonal disorders. Pathogenic variants in genes on the sex chromosomes have key roles in spermatogenic failure. The co-occurrence of azoospermia and congenital cataracts ranges between 1 in 165,000 and 1 in 500,000. Our 28-year-old patient with normal intelligence and abnormally shaped teeth presented with both disorders. A microarray revealed a microdeletion at Xp23.13 with a whole NHS gene deletion as well as a contiguous deletion of two other genes [SCML1 and RAI2]. This observation represents the first report of non-obstructive azoospermia with congenital cataracts and a contiguous deletion of the SCML1 gene, a transcript of which is exclusively expressed in the testis. SCML1 is the putative culprit gene, which requires functional study or animal experiments. Our analysis of 60 known spermatogenesis failure-related genes by whole-exome sequencing revealed no other candidate. The Nance-Horan syndrome due to pathogenic variants in the NHS gene at Xp23.13 including whole gene deletion does not have azoospermia as a feature. Our report adds to the completeness of genetic counseling for an individual with azoospermia and congenital cataracts.
\end{abstract}

Keywords Azoospermia $\cdot$ Congenital cataracts $\cdot$ Spermatogenesis $\cdot$ Microdeletion $\cdot$ SCML1 gene

\section{Introduction}

An estimated 30 million males worldwide are infertile. ${ }^{1}$ In up to $72 \%$ of these males, no cause has been determined. ${ }^{2}$ More than 2300 genes are involved in spermatogenesis in the mouse. ${ }^{3}$ A similar number is likely in man. Nonobstructive azoospermia accounts for $10-15 \%$ of male

Aubrey Milunsky

amilunsky@chginc.org

1 Center for Human Genetics, 840 Memorial Dr, Ste 101, Cambridge, MA 02139, USA

2 Department of Obstetrics and Gynecology, Tufts University School of Medicine, Boston, MA, USA

3 Department of Human Genetics, Yale University School of Medicine, New Haven, CT, USA

4 Department of Urology, Boston Medical Center and Boston University School of Medicine, Boston, MA, USA infertility, resulting in $60 \%$ of all cases of azoospermia, affecting about $1 \%$ of the male population. ${ }^{1,4}$ About $30 \%$ of these cases are due to $\mathrm{Y}$ chromosome microdeletions, chromosomal abnormalities, or hormonal disorders. ${ }^{4-6}$ Autosomal gene variants are primarily involved in hormone-based hypogonadism, oligospermia, teratozoospermia, congenital obstructive azoospermia, and familial spermatogenic abnormalities. Pathogenic variants in genes on the sex chromosomes, however, have key roles in spermatogenic failure.

Congenital cataracts occur with an estimated frequency of 1-3/10,000 live births. ${ }^{7} 8$ Estimates suggest that between 22 and $40 \%$ of congenital cataracts are due to single-gene mutations. Pathogenic variants in at least 40 genes are known for congenital cataracts ${ }^{9}$ [CAT-MAP; http://cat-map.wustl.edu/]. However, the co-occurrence of congenital cataracts and azoospermia in association with an $\mathrm{X}$ chromosome microdeletion has not been reported. Here, we report a whole NHS gene (OMIM: 300457) deletion with contiguous deletion of 2 genes: SCML1 (OMIM: 300227) and RAI2 (OMIM: 300217). 


\section{Material and methods}

\section{Editorial policies and ethical considerations}

This study was approved by the Ethics Committee at the Center for Human Genetics. Informed consent was obtained from the patient for these DNA studies.

The patient, a 28-year-old male, first presented with concern about infertility. His 29-year-old wife had a son in a previous union, but together they failed to conceive for more than 1 year. Their hope and plan was for IVF. His past medical history included surgery for congenital cataracts.

His family history revealed no member with cataracts. He has a sister with arthritis, a father with diabetes type II, hypothyroidism, and heart disease. One deceased paternal uncle had diabetes type II, hypertension, and Parkinson's disease. His mother had five siblings, none of whom had cataracts or significant illnesses and whose children were said to be healthy. His wife, one of the 10 children, is the daughter of his paternal aunt. All are said to be healthy except for one with diabetes type II. His wife is Palestinian, and he is of Lebanese decent.

Physically, he was intelligent, tall, burly, and mildly dysmorphic, with low-set ears and abnormally shaped small teeth. Nystagmus, microcornea, strabismus, and right exotropia were evident. He was being treated for glaucoma. He was well-virilized and his external genital anatomy was normal. Testes were slightly small and measured approximately $12 \mathrm{cc}^{3}$ bilaterally without mass, area of induration, or tenderness. His vasa differentia and epididymis were normal to palpation, and there was no varicocele or spermatocele on either side. Multiple centrifuged semen analyses demonstrated slightly low-volume azoospermia. Endocrine studies revealed follicle-stimulating hormone 7.1 [FSH: $0.7-10.8 \mathrm{mlU} / \mathrm{ml}$ ] consistent with spermatogenic compromise; luteinizing hormone 6.2 [LH; 1.2-10.6 $\mathrm{mlU} / \mathrm{ml}$ ]; and testosterone 239 [T; 241-827 ng/dl]. Based upon these collective findings suggestive of non-obstructive azoospermia, a Y-microdeletion assay and a karyotype were obtained.

The only therapeutic option for the couple was microsurgical testis sperm extraction (mTESE) in hopes of finding spermatozoa scattered about the testicular parenchyma with the intent of cryopreserving them for future use as a sperm source in conjunction with intracytoplasmic sperm injections (ICSI). If an underlying genetic etiology and/or syndrome responsible for the phenotypic features described above could be determined, the couple was prepared to employ preimplantation genetic diagnosis on embryos created and transfer only those that would not vertically transmit this genomic aberration to any conceived offspring. In carefully considering the sequence of events, the couple decided to proceed along with mTESE while awaiting the final genetic analysis outcome. Exhaustive mTESE was accomplished bilaterally, and, unfortunately, no spermatozoa could be detected. Histological findings (Fig. 1) showed a predominant pattern of spermatocytic maturation arrest $(90 \%$; seminiferous tubules filled with spermatogonia and spermatocytes but no spermatids, reflective of a block in meiotic progress) and germ cell aplasia (10\%; seminiferous tubules with a complete absence of germ cells, only Sertoli cells lining the interior basement membrane of the tubule).

From a peripheral blood sample, the following genetic studies were conducted: chromosomal analysis, Ymicrodeletion studies, SNP 6.0 microarray, analysis of the most common 116 pathogenic variants in the cystic fibrosis gene [CFTR], Sanger sequencing of the NR5A1 (OMIM: 184757) gene; Sanger sequencing and multiplex ligationdependent probe amplification (MLPA) of 2-9 exons of the SYCP3 (OMIM: 604754) gene; next-generation sequencing $\geq 50 \mathrm{X}$ depth of 40 known cataract genes, and MLPA were included, with sequencing only of the CTDP1 (OMIM: 604927) gene.

Whole-exome sequencing (WES) was then performed at the Yale Center for Genomic Analysis using IDT xGen target capture kit, followed by paired-end sequencing on Illumina NovaSeq 6000 platform with $100 \mathrm{bp}$ read length. The sequencing reads were aligned onto the GRCh37/hg19 reference genome using Burrow-Wheeler Aligner-MEM (BWA-MEM) program. ${ }^{10}$ Single nucleotide changes and small indels were called using GATK Best Practices workflow ${ }^{11-13}$ and then were annotated with ANNOVAR. ${ }^{14}$

Variants were kept if they are of high confidence (quality score recalibration [VQSR] "PASS", read depth [DP] $\geq 8$, genotype quality $[\mathrm{GQ}]$ score $\geq 20$, and mapping quality [MQ] score $\geq 40$ ). Variants were then prioritized if they are rare (minor allele frequency $[\mathrm{MAF}]<5 \times 10^{-5}$ in both Genome Aggregation Database [gnomAD v2.0] whole exome and whole genome databases). ${ }^{15}$ Furthermore, variants were kept if they have damaging effect on protein structure, including loss-of-function variants (canonical splice-site, frameshift insertion/deletion, stop-gain, stop-loss) and deleterious missense variants (defined by MetaSVM "D"). ${ }^{16}$ In silico visual inspections were performed on all the variants passing the above filters.

\section{Results}

Analysis of the most common 116 mutations in the CFTR gene revealed no pathogenic variants, with the IVS8-polyT tract being $7 \mathrm{~T} / 7 \mathrm{~T}$. Sequencing and MLPA analysis of the NR5A1/SF1 gene revealed no abnormality in exons 2-7, which performed to determine a $46 \mathrm{XY}$ disorder of sex development with or without adrenal insufficiency or to explain severe spermatogenic failure. 


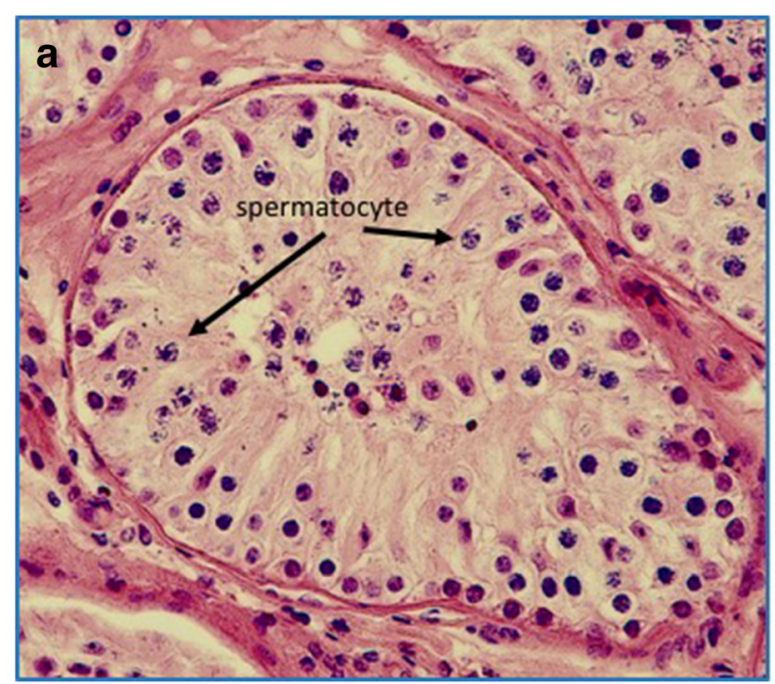

Fig. 1 (a) Cross-section of a single seminiferous tubule with a pattern of spermatocytic maturation arrest. Spermatogonia and spermatocytes are numerous, but no haploid spermatids are seen. (b) Cross-section of a

Sequencing and MLPA of 2-9 exons of the SYCP3 gene revealed no pathogenic variants, which have been reported in association with non-obstructive azoospermia and recurrent pregnancy loss in females.

Sequencing and MLPA analysis of the autosomal recessive TDRD7 gene (OMIM: 611258) showed no pathogenic variants. Loss-of-function pathogenic variants in this gene have been reported in consanguineous patients with congenital cataracts and azoospermia. ${ }^{17}$

A panel of 40 genes related to cataracts was sequenced and no pathogenic variant found (Supplementary Table 1). ${ }^{9}$

The karyotype was normal. The SNP microarray showed a $721 \mathrm{~kb}$ deletion at Xp23.13 [ChrX: 17,409,227-18,130,251; hg19]. This deleted region is the location of 3 OMIM genes including SCML1 [OMIM: 300227], NHS [OMIM: 300456], and RAI2 [OMIM: 300217].

For whole-exome sequencing, the average depth was 69.6, with $97.5 \% 20 \times$ target base coverage. Nineteen rare (MAF $\leq$ $5 \times 10^{-5}$ from gnomAD) damaging variants that are either loss-of-function or deleterious missense defined by MetaSVM were called. None of the variants are from 60 known spermatogenesis failure-related genes ${ }^{2}$ (Supplementary Table 2$)^{2}$ or are reported as pathogenic in ClinVar.

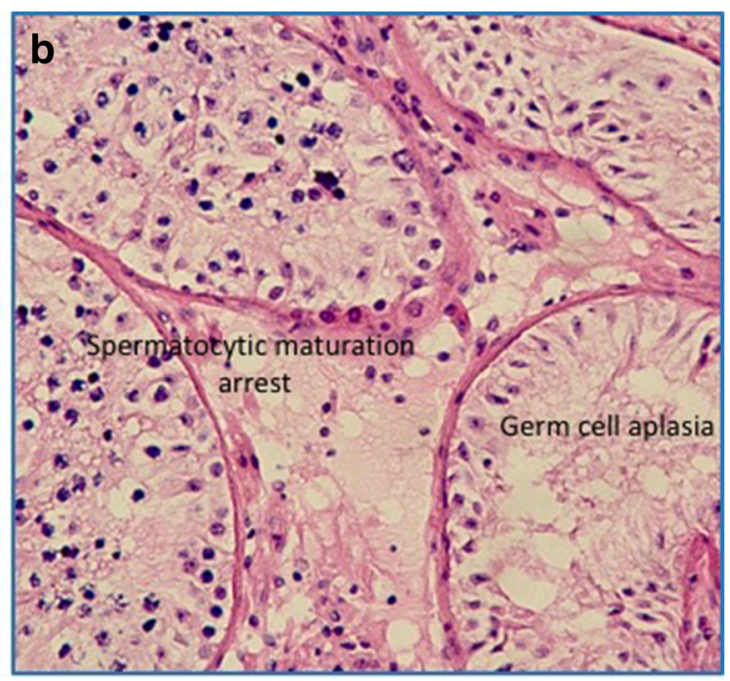

few adjacent seminiferous tubules displaying spermatocytic maturation arrest in the two on the left-hand side and germ cell aplasia in the two on the right-hand side

\section{Discussion}

The rare X-linked Nance-Horan syndrome ${ }^{18}$ is characterized by a host of variable signs that include congenital cataracts, dental abnormalities, intellectual disability in some, dysmorphism, microcornea, and microphthalmia. Our patient stated that his mother had neither cataracts nor any other features of this syndrome. We were unable to obtain a blood sample from her. Azoospermia is, however, not a feature of this syndrome. Missense or frameshift pathogenic variants or partial or whole NHS gene deletions are well recognized ${ }^{19-21}$, including contiguous deletion of the SCML1 and RA12 genes [Fig. 2]. Despite the published speculation that the SCML1 gene was involved in spermatogenesis ${ }^{20}$, we undertook whole-exome sequencing. The special focus was on all known azoospermia genes to be reasonably certain that no other hidden candidate could be implicated in our patient. Consequently, with no other culprit gene determined, this report is the first to record the Xp23.13 microdeletion with whole NHS gene deletion resulting in cataracts and azoospermia, highly likely due to contiguous deletion of the SCML1 gene. Immunohistochemical data indicate that this gene is preferentially expressed in germ stem cells in the testes and hence involved in spermatogenesis. The SCML1 gene has 5

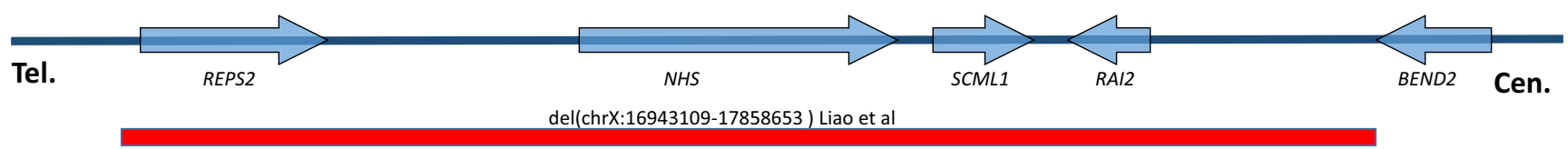

del(chrX 17733948 17904576) Accogli et al

del(chrX:17409227-18170251) This publication

Fig. 2 Schematic illustration of the Xp22.13 microdeletion involving NHS, SCML1, and RAI2 genes in comparison with previous reported cases ${ }^{19}$, 20 
protein-coding transcripts, one of which, ENST00000380043, is exclusively expressed in the testis.

Two other reports with similar microdeletions [Fig. 2] ${ }^{19,20}$ in which the SCML1 gene was deleted had no semen samples. Liao et al. ${ }^{19}$ speculated that the SCML1 deletion could have reproductive consequences. The RAI2 gene was also deleted in our patient and in the other two reports. ${ }^{19,20}$ This gene is involved in the regulation of cell differentiation and embryonal development. No definitive role or consequence for the deletion of this gene can be ascribed in our patient.

It is ironic that the $\mathrm{X}$ chromosome has a major role in male infertility and spermatogenesis. This effect is reflected by the occurrence of $\mathrm{X}$ chromosome aneuploidy [Klinefelter syndrome], translocations involving the $\mathrm{X}$ chromosome, copy number variations [CNVs] especially represented by $\mathrm{X}$ chromosome microdeletions, and pathogenic variants in the $\mathrm{X}$ chromosome. ${ }^{1,2,22}$ Of the estimated $>2300$ genes involved in spermatogenesis ${ }^{23}$, about $25 \%$ cause or contribute to azoospermia. In only about a fifth of patients with azoospermia is a genetic cause currently determined.

Azoospermia and congenital cataracts have been reported in the oculocerebrorenal syndrome of Lowe. ${ }^{24}$ This syndrome without any semblance to our patient is characterized by manifestations that include intellectual disability, proximal renal tubular dysfunction, severe hypotonia, buphthalmos, and dysmorphism. Pathogenic variants in the OCRL gene located on Xq26.1 account for this rare syndrome.

Azoospermia and early cataracts were also reported in an Algerian family with apparently an X-linked hereditary multi-system disorder associated with the moyamoya Syndrome. ${ }^{25}$ Haplotype linking of the maternal lineage supported X-linked inheritance in the five affected males. Signs included acute neurologic manifestations between 4 and 32 years of age, hypergonadotrophic hypogonadism, azoospermia, cataract, short stature, premature gray hair, dysmorphism, cardiomyopathy, and partial growth hormone deficiency. ${ }^{25}$ Again, these features were not consistent with those affecting our patient.

Prenatal diagnosis of fetal cataracts has been made by ultrasound study in the second trimester of pregnancy. ${ }^{26}$ Subsequent sequencing revealed a frameshift mutation (3908dl11bp) in the NHS gene. Now we know for genetic counseling and/or prenatal diagnosis that a contiguous deletion that includes the SCML1 gene in a male is highly likely to be associated with azoospermia. ${ }^{27}$

\section{Compliance with ethical standards}

Conflict of interest The authors declare that they have no conflicts of interest.

\section{References}

1. Colaco S, Modi D. Genetics of the human Y chromosome and its association with male infertility. Reprod Biol Endocrinol. 2018;16(1):14.

2. Cannarella R, Condorelli RA, Duca Y, La Vignera S, Calogero AE. New insights into the genetics of spermatogenic failure: a review of the literature. Hum Genet. 2019;138(2):125-40.

3. Schultz N, Hamra FK, Garbers DL. A multitude of genes expressed solely in meiotic or postmeiotic spermatogenic cells offers a myriad of contraceptive targets. Proc Natl Acad Sci. 2003;100(21):12201-6.

4. Cooper TG, Noonan E, Von Eckardstein S, Auger J, Baker HW, et al. World Health Organization reference values for human semen characteristics. Hum Reprod Update. 2010;16(3):231-45.

5. Agarwal A, Mulgund A, Hamada A, Chyatte MR. A unique view on male infertility around the globe. Reprod Biol Endocrinol. 2015;13(1):37.

6. Jarow JP, Sharlip ID, Belker AM, Lipshultz LI, Sigman M, Thomas AJ, et al. Best practice policies for male infertility. J Urol. 2002;167(5):2138-44.

7. Daien V, Le Pape A, Heve D, Villain M, Gignac DB. Incidence and characteristics of congenital cataract surgery in France from 2010 to 2012: the EPISAFE program. Ophthalmic Res. 2017;58(2):114-6.

8. Magnusson G, Haargaard B, Basit S, Lundvall A, Nyström A, Rosensvärd A, et al. The Paediatric Cataract Register (PECARE): an overview of operated childhood cataract in Sweden and Denmark. Acta Ophthalmol. 2018;96(1):51-5.

9. Shiels A, Hejtmancik JF. Mutations and mechanisms in congenital and age-related cataracts. Exp Eye Res. 2017;156:95-102.

10. Li H, Durbin R. Fast and accurate short read alignment with burrows-wheeler transform. Bioinformatics. 2009;25(14):1754-60.

11. McKenna A, Hanna M, Banks E, Sivachenko A, Cibulskis K, Kernytsky A, et al. The genome analysis toolkit: a MapReduce framework for analyzing next-generation DNA sequencing data. Genome Res. 2010;20(9):1297-303.

12. DePristo MA, Banks E, Poplin R, Garimella KV, Maguire JR, Hartl $\mathrm{C}$, et al. A framework for variation discovery and genotyping using next-generation DNA sequencing data. Nat Genet. 2011;43(5): 491-8.

13. Van der Auwera GA, Carneiro MO, Hartl C, et al. From FastQ data to high confidence variant calls: the genome analysis toolkit best practices pipeline. Curr Protoc Bioinformatics. 2013;43(1):11-0.

14. Wang K, Li M, Hakonarson H. ANNOVAR: functional annotation of genetic variants from high-throughput sequencing data. Nucleic Acids Res. 2010;38(16):e164.

15. Lek M, Karczewski KJ, Minikel EV, et al. Analysis of proteincoding genetic variation in 60,706 humans. Nature. 536(7616): 285-91.

16. Dong C, Wei P, Jian X, Gibbs R, Boerwinkle E, Wang K, et al. Comparison and integration of deleteriousness prediction methods for nonsynonymous SNVs in whole exome sequencing studies. Hum Mol Genet. 2015;24(8):2125-37.

17. Tan YQ, Tu C, Meng L, Yuan S, Sjaarda C, Luo A, et al. Loss-offunction mutations in TDRD7 lead to a rare novel syndrome combining congenital cataract and nonobstructive azoospermia in humans. Genetics in Medicine. 2019;21(5):1209-17.

18. Brooks SP, Ebenezer ND, Poopalasundaram S, Lehmann OJ, Moore AT, Hardcastle AJ. Identification of the gene for NanceHoran syndrome (NHS). J Med Genet. 2004;41(10):768-71.

19. Liao HM, Niu DM, Chen YJ, Fang JS, Chen SJ, Chen CH. Identification of a microdeletion at Xp22.13 in a Taiwanese family presenting with Nance-Horan syndrome. Journal of Human Genetics. 2011;56(1):8. 
20. Accogli A, Traverso M, Madia F, Bellini T, Vari MS, Pinto F, et al. A novel Xp22. 13 microdeletion in Nance-Horan syndrome. Birth Defects Research. 2017;109(11):866-8.

21. Ling C, Sui R, Yao F, Wu Z, Zhang X, Zhang S. Whole exome sequencing identified a novel truncation mutation in the NHS gene associated with Nance-Horan syndrome. BMC Medical Genetics. 2019;20(1):14.

22. Mitchell MJ, Metzler-Guillemain C, Toure A, Coutton C, Arnoult C, Ray PF. Single gene defects leading to sperm quantitative anomalies. Clin Genet. 2017;91(2):208-16.

23. Mueller JL, Mahadevaiah SK, Park PJ, Warburton PE, Page DC, Turner JMA. The mouse $\mathrm{X}$ chromosome is enriched for multicopy testis genes showing postmeiotic expression. Nat Genet. 2008;40(6):794-9.

24. Bökenkamp A, Ludwig M. The oculocerebrorenal syndrome of Lowe: an update. Pediatr Nephrol. 2016;31(12):2201-12.
25. Hervé D, Touraine P, Verloes A, Miskinyte S, Krivosic V, Logeart $\mathrm{D}$, et al. A hereditary moyamoya syndrome with multisystemic manifestations. Neurology. 2010;75(3):259-64.

26. Reches A, Yaron Y, Burdon K, Crystal-Shalit O, Kidron D, Malcov $\mathrm{M}$, et al. Prenatal detection of congenital bilateral cataract leading to the diagnosis of Nance-Horan syndrome in the extended family. Prenatal Diagnosis: Published in Affiliation with the International Society for Prenatal Diagnosis. 2007;27(7):662-4.

27. Wu HH, Su B. Adaptive evolution of SCMLI in primates, a gene involved in male reproduction. BMC Evol Biol. 2008;8(1):192.

Publisher's note Springer Nature remains neutral with regard to jurisdictional claims in published maps and institutional affiliations. 